\title{
Longitudinal Stability Augmentation of Seaplanes in Planing
}

\author{
Keiichi Ito*† and Tom Dhaene ${ }^{\ddagger}$ \\ Ghent University - iMinds, Ledeberg - Ghent, 9050, Belgium \\ Yoshiaki Hirakawa, and Tsugukiyo Hirayama \\ Yokohama National University, Yokohama, Kanagawa, 240-8501, Japan \\ Tatsumi Sakurai $\|$ \\ Hiyoh Aircraft Manufacturing and Development, Shinagawa, Tokyo, 142-0041, Japan
}

The towing tank experiments conducted at the Yokohama National University from November 30 to December 9 in 2005, suggested a new way of suppressing a dangerous coupled motion between heave and pitch called porpoising. This research develops on the observations made in the experiments and conducts numerical simulations to further investigate the parametric design space. Two Linear-Time-Invariant models were developed: rigid-body planing craft (conventional float planes or flying boats) and flexibly supported planing craft. The latter can simulate the new method found in the experiments for suppressing porpoising. In this study, the stability of the oscillatory motions was analyzed to see the effect of design variables to the inception of porpoising. The parametric study of flexibly supported float planes in the context of porpoising is a new contribution in the conceptual design of seaplanes.

\footnotetext{
${ }^{*}$ Researcher, Department of Information Technology (INTEC), Gaston Crommenlaan 8 bus 201, 9050 Ledeberg - Ghent, Belgium, and AIAA Student Member.

${ }^{\dagger}$ Technical Specialist, Noesis Solutions, Gaston Geenslaan 11 B4, 3001 Leuven, Belgium

${ }^{\ddagger}$ Professor, Department of Information Technology (INTEC), Gaston Crommenlaan 8 bus 201, 9050 Ledeberg - Ghent, Belgium

$\S$ Associate Professor, Department of System Integration, 79-5, Tokiwadai, Hodogayaku, Yokohama 2408501, Japan

『Professor Emeritus, Department of System Integration, 79-5, Tokiwadai, Hodogayaku, Yokohama 2408501, Japan

"Director, 1-7-1, Togoshi, Shinagawaku, Tokyo 142-0041, Japan
} 


\section{Nomenclature}

$\beta \quad$ Deadrise angle in [radians]

$\beta_{\text {deg }} \quad$ Deadrise angle in [degrees]

$\epsilon \quad$ Thrust line angle with respect to the keel line (positive upwards) in [radians]

$\eta_{3}, \eta_{5}$ Displacement in heave and pitch respectively from the inertial coordinate $x_{b}, z_{b}$

$\lambda_{0} \quad \lambda_{W}$ at the static equilibrium position

$\lambda_{W} \quad$ Mean wetted length to beam ratio, $0.5\left(L_{K}+L_{C}\right) / B$

$\Re(\sigma)_{\max }$ Maximum real part of the eigenvalues of a matrix $\mathbf{K}$ as in $\dot{\mathbf{x}}=\mathbf{K x}$, unit in $[1 / s]$

$\rho \quad$ Water density in $\left[\mathrm{kg} / \mathrm{m}^{3}\right]$

$\tau \quad$ Trim angle of planing area in [radians]

$\tau_{\text {deg }} \quad$ Trim angle of planing area in [degrees]

$\xi_{3}, \xi_{5}$ Displacement in heave and pitch respectively from the inertial coordinate $x_{a}, z_{a}$

$a \quad$ Distance between CG and $R_{v}$ (positive when pitch-down moment results) in [ $\left.\mathrm{m}\right]$

$a_{33}$ Two-dimensional added mass of a wedge plunging into water in $[\mathrm{kg} / \mathrm{m}]$

$A_{i j} \quad$ Hydrodynamic added mass/moment of inertia in the direction of $i$ due to the motion in the direction of $j$

$A H R$ Average hull roughness in $[\mu m]$

$B \quad$ Beam length (i.e. width) of a float in $[m]$

$B_{i j} \quad$ Hydrodynamic damping coefficient in the direction of $i$ due to the motion in the direction of $j$

c Pitching moment arm of hydrodynamic resultant pressure force $N$ with respect to CG (positive when pitch-down moment results), $l_{c g}-l_{p}$

$C_{F} \quad$ Friction coefficient

$c_{f, b} \quad$ Damping coefficients in the flexible support (front and back) in $[N \mathrm{~s} / \mathrm{m}]$

$C_{i j} \quad$ Hydrodynamic restoring force/moment coefficient in the direction of $i$ due to the motion in the direction of $j$

$C_{L_{0}} \quad$ Lift coefficient for zero deadrise angle $(\beta=0$ [rad.])

$C_{L_{\beta}} \quad$ Lift coefficient

$d \quad$ Draft in $[m]$

$f \quad$ Thrust line distance from CG (positive when pitch-up moment results) in $[m]$

$F_{L_{0}} \quad$ Lift force for zero deadrise angle $(\beta=0$ [rad.])

$F_{L_{\beta}} \quad$ Lift force

$F_{n_{B}} \quad$ Froude number based on the beam length defined as $U / \sqrt{g B}$, where $g$ is gravitational acceleration

$I_{A} \quad$ Pitching mass moment of inertia of the aircraft without the floats for the flexiblesupport model in $\left[\mathrm{kg} \mathrm{m}^{2}\right]$ 
$I_{B} \quad$ Pitching mass moment of inertia of the floats for the flexible-support model in $\left[\mathrm{kg} \mathrm{m}^{2}\right]$

$I_{55} \quad$ Pitching mass moment of inertia of rigidly supported seaplane/model in $\left[\mathrm{kg} \mathrm{m}^{2}\right]$

$k_{f, b} \quad$ Spring constants in the flexible support (front and back) in $[N / m]$

$L_{C} \quad$ Wetted chine length measured from the step in $[\mathrm{m}]$

$L_{K} \quad$ Wetted keel length measured from the step in $[m]$

$l_{p} \quad$ Distance measured along the keel from the step to the center of hydrodynamic pressure in $[m]$

$l_{A f, A b}$ Attachment locations of flexible supports on the aircraft relative to center of gravity of the aircraft in $[m]$

$l_{B f, B b}$ Attachment locations of flexible supports on the floats relative to center of gravity of the floats in $[m]$

$l_{c g} \quad$ Longitudinal distance of center of gravity along the keel line measured from the step or transom in $[m]$

$M \quad$ Mass of the aircraft for the rigid body model in $[\mathrm{kg}]$

$m_{A} \quad$ Mass of the aircraft for the flexible-support model in $[\mathrm{kg}]$

$m_{B} \quad$ Mass of the float for the flexible-support model in $[\mathrm{kg}]$

$N \quad$ Resultant hydrodynamic pressure force acting on the planing surface in $[N]$

$N_{f} \quad$ Number simulation function calls

$N_{s} \quad$ Number of simulation function calls that satisfied the stability criteria

$R_{n} \quad$ Reynolds number $U L_{K} / \nu$, where $\nu$ is the kinematic viscocity of water

$R_{v} \quad$ Frictional force acting on the planing hull assumed to act parallel to keel line in $[N]$

$T \quad$ Thrust force in $[N]$

$t \quad$ Time in $[s]$

$U \quad$ Planing speed in $[\mathrm{m} / \mathrm{s}]$

$V \quad$ Vertical velocity with which the hull impacts the water surface, $U \tan \tau$

$v c g \quad$ Vertical distance of CG from the keel line in $[m]$

$x_{a}, z_{a}$ Inertial coordinate moving with the aircraft's CG's equilibrium position without floats, $x_{a}$ pointing horizontally to the stern, $z_{a}$ pointing vertically upward

$x_{b}, z_{b}$ Inertial coordinate moving with the aircraft's CG's equilibrium position when the supports are rigid and moving along the floats' CG's equilibrium position when the supports are flexible, $x_{b}$ pointing horizontally to the stern, $z_{b}$ pointing vertically upward

$z_{\max }$ Height above the undisturbed water surface where the maximum pressure on the V-shaped hull happens due to its impact to the water 


\section{Introduction}

EAPLANES and their amphibian versions have been largely a neglected type of aircraft in $S$ recent aviation except for very specific missions and in limited geographic regions. This is due to higher maintenance costs, less payload, and lower operational reliability (high waves are an additional weather hazard) compared to land based aircraft. However, recent technological advances in materials and computational capabilities along with macro-economic and ecological considerations may render this type of aircraft interesting. Point-to-point operation in coastal area could alleviate traffic congestion in urban airports and make remote islands more accessible. This in turn should help more balanced economic growth and better emergency services in smaller cities and rural areas. This paper addresses one of the drawbacks of seaplanes called porpoising which is a dynamic instability in planing seaplanes and high-speed boats. ${ }^{1}$

Porpoising is a coupled oscillatory motion between heaving and pitching that can manifest when seaplanes are travelling on water at planing speed (Figure 1). This motion may become unstable and can pose significant risk to the safe operation of waterborne aircraft. Traditionally, the rules of thumb in designing of hulls and physical experiments ${ }^{2-5}$ (combined with pilot training ${ }^{6,7}$ ) have been the methods of mitigating the risk. However, the phenomenon is poorly understood and sufficient parametric studies applicable to seaplanes have not appeared in the literature. Current research aims to fill this gap. The objective is to effectively mitigate or eliminate porpoising by design.

Towing tank experiments ${ }^{8}$ showed that the moving center of gravity aft, or employing flexible supports (between the aircraft and the floats) comparable to those of a car could improve the stability of the planing craft (Figure 2). To understand these observations, Linear-Time-Invariant models were constructed and the stability of oscillatory motions was studied. The numerical models were coherent with the experiments and two major design question were answered, namely 1) the appropriate direction to move the center of gravity when porpoising is a problem, ${ }^{9} 2$ ) whether flexible supports suppress porpoising globally or under certain conditions.

In the literature, most of the work investigating longitudinal stability of planing seaplanes is experimental. A large portion of them was conducted before the prevalence of fast personal computers (i.e., before the '70s). Parametric investigation of porpoising behavior based on numerical simulations and investigation of flexible supports for mitigation of porpoising are two contributions of this work. 


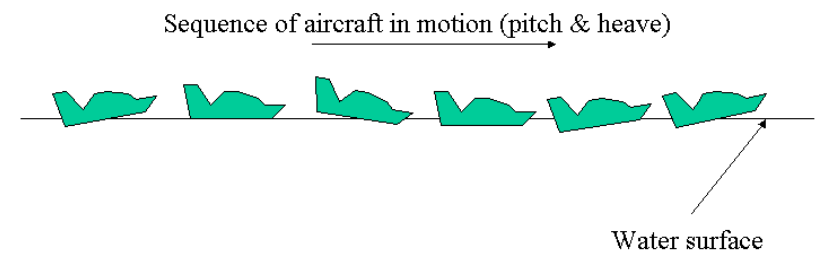

Figure 1: Schematic of porpoising

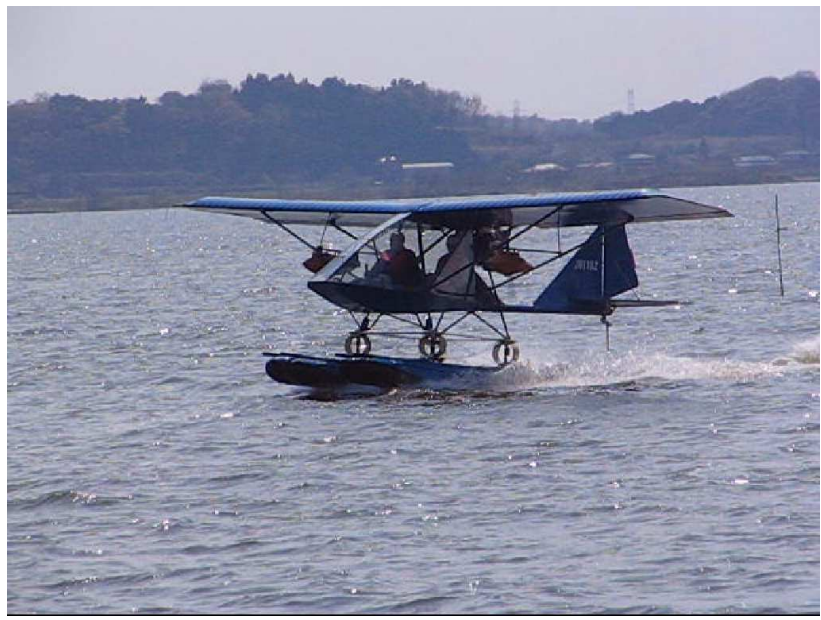

Figure 2: An implementation of the Flexible Support System in an Ultra Light Plane

\section{Methods}

The first step in this study was to numerically recreate at least qualitatively the observation made in the towing tank experiments. Particularly, the objective here is to confirm that the inception of porpoising occurs at approximately $5 \mathrm{~m} / \mathrm{s}$ of towing speed with the original CG location, and that by moving the CG aft stabilizes the towed model. Then, observe numerically that the flexible support "stabilizes" the towed model. The second step was to explore different designs by varying parameters in the numerical model. The towed model consisted of a pair of floats in catamaran configuration and a frame on top of it (Figure 3). The frame has adjustable weights to roughly simulate the inertia characteristics of the aircraft that the float was designed for, which is a 1/3 scale Piper Cub. By changing the location of weights, one can also move the location of the CG backward or forward. In the following, the numerical model of the conventional rigid case and then the flexibly supported 
case are described.

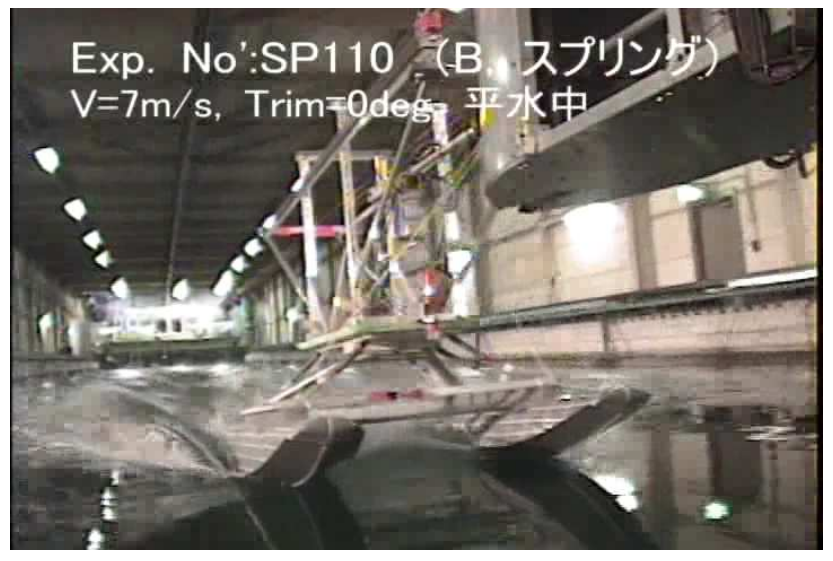

Figure 3: Experiment with the flexible support at the towing tank of Yokohama National University

For the numerical analysis, the catamaran configuration was replaced by a mono-hull representation of prismatic hull as shown in Figure 4. The transom location in this figure corresponds to the step location in the actual float. The afterbody of the float is neglected in the modelling and so is the curved front portion of the forebody of the float. The dynamic stability was computed using small perturbation analysis as presented in Faltinsen. ${ }^{10}$ The coordinate systems employed are shown in Figure 6. For the rigidly supported case, the inertial coordinate system $x_{b}, z_{b}$ was set to move along with the towed craft, its origin coincides with the equilibrium position of the craft's center of gravity. The $x$ axes point to the stern of the craft. For the flexibly supported case, separate inertial coordinate systems that move along the craft were employed for above the support $x_{a}, z_{a}$ and the float $x_{b}, z_{b}$. These are an approximate way to represent the dynamics (for the frame has surge component of motion that are neglected) but were done in order to facilitate the analysis. The linear system of equation for the rigidly supported case is equation (1), and for the flexibly supported case, equation (2). Added mass $A_{i j}$, damping force coefficient $B_{i j}$, and the restoring force coefficient $C_{i j}$ were formulated according to chapter 8 and 9 of Faltinsen's book. ${ }^{10}$ A cursory description of how $A_{i j}, B_{i j}$, and $C_{i j}$ are computed is given in the appendix. The numbers in subscripts $i, j \in\{3,5\}$ denote heaving ${ }_{3}$ and pitching ${ }_{5}$ respectively. The first subscript ${ }_{i}$ refers to the resulting force or moment direction and the second subscript ${ }_{j}$ refers to the motion causing the force or moment. For example, $C_{35}$ refers to the heaving force coefficient due to pitching motion. One can also find relevant information on the hydrodynamic forces for planing crafts in. ${ }^{9,11-13}$

The flexibly supported case contains additional parameters on the characteristics of the support, namely the spring constants $k_{f}, k_{b}$ and the damping coefficients $c_{f}, c_{b}$. The subscripts denote their locations: ${ }_{f}$ for front and ${ }_{b}$ for back. Likewise, the attachment locations 


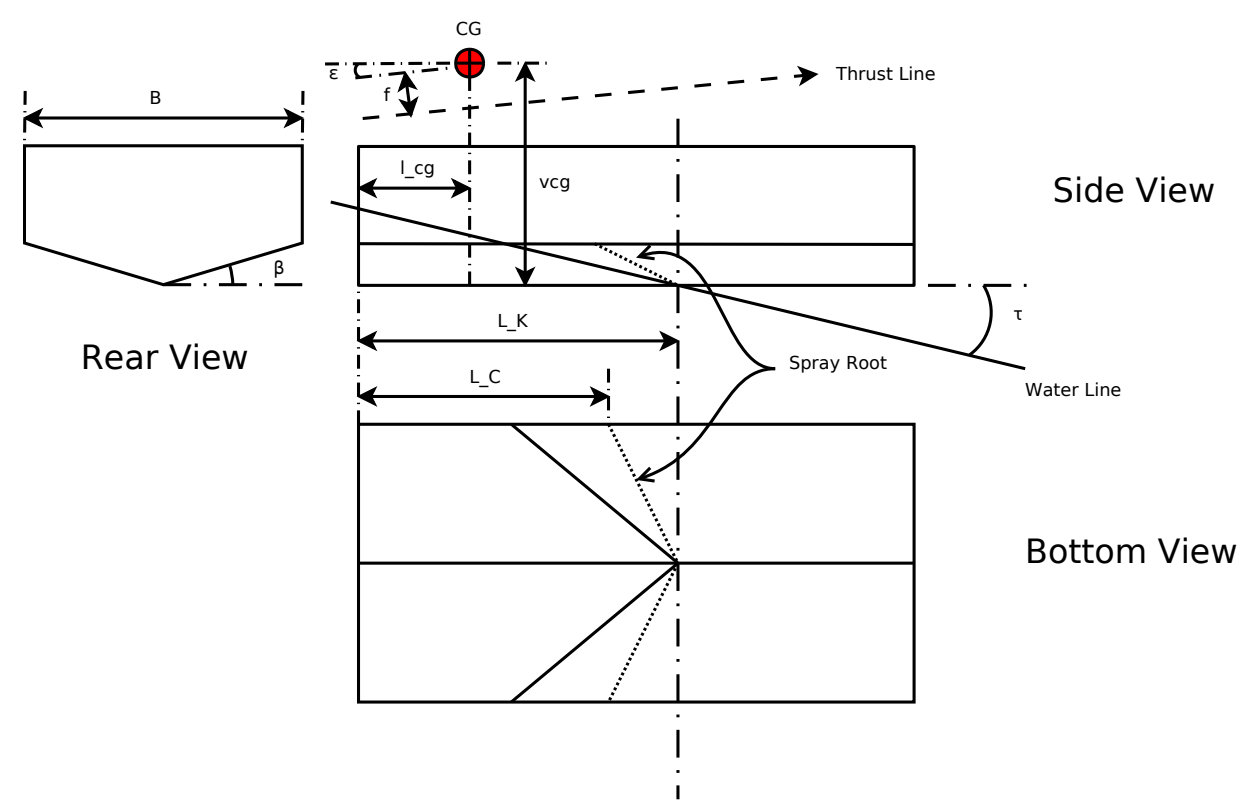

Figure 4: Diagram of planing hull cut-out.

relative to center of gravity are denoted $l_{A f}, l_{A b}$ for the front and back attachment point on the aircraft side respectively, and $l_{B f}, l_{B b}$ on the float side. These parameters are visualized in Figure 5. We have kept the attachment points fixed and varied the spring constants and damping coefficients in our numerical simulations.

In the rigidly supported case, we also used Self-Organizing Map Based Adaptive Sampling $(\text { SOMBAS })^{14,15}$ to search for stable designs. SOMBAS is suitable for the task of searching for multiple and diverse solutions satisfying certain objective conditions. We searched for designs with negative values in maximum real part of the eigenvalues. For the two-designvariable case, we use the longitudinal distance of CG along the keel line $l_{c g}$ measured from the step or transom, and vertical distance of CG from the keel line $v c g$. For the seven-designvariable case, we use the beam length $B$, the deadrise angle $\beta_{\text {deg }}$, the pitching moment of inertia $I_{55}$, the thrust line distance $f$ from CG (positive when pitch-up moment results) and the thrust line angle with respect to the keel line (positive upwards) $\epsilon$. Figure 4 shows a diagram describing the design variables except the inertial variable $I_{55}$. 


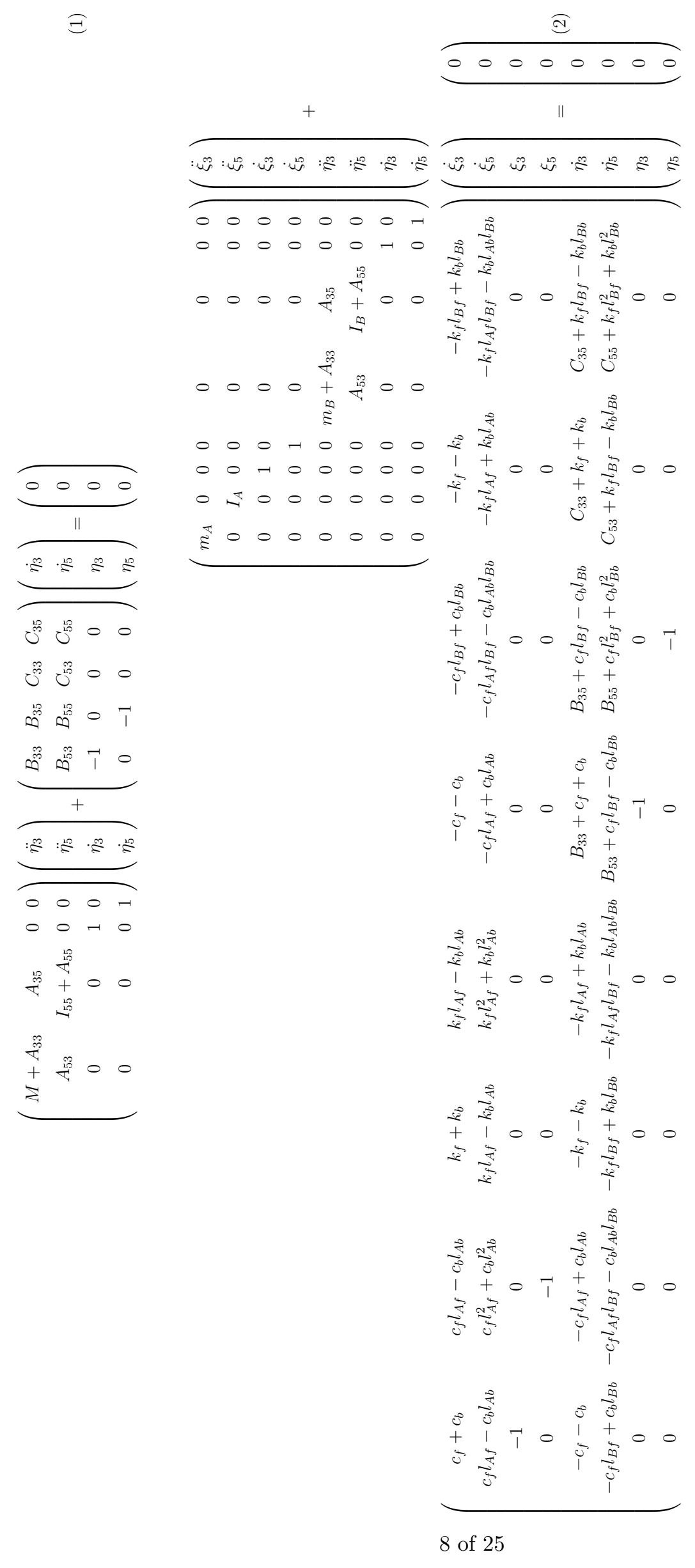




\section{Results}

Figure 7 - Figure 9 and Figure 14 - Figure 15 are for the rigidly supported case and Figure 10 - Figure 13 are for the flexibly supported case. Nominal conditions for the calculation are as following unless otherwise specified: the planing speed $U=6.0[\mathrm{~m} / \mathrm{s}]$, the mass of craft for the rigid case $M=16.18[\mathrm{~kg}]$ and for the flexible case $m_{A}=10.79[\mathrm{~kg}]$ (aircraft), $m_{B}=5.39[\mathrm{~kg}]$ (float), the beam length $B=0.2[\mathrm{~m}]$, the moment of inertia for the rigid case $I_{55}=5.981\left[\mathrm{~kg} \mathrm{~m}^{2}\right]$, for the flexible case $I_{A}=4.351\left[\mathrm{~kg} \mathrm{~m}^{2}\right]$ (aircraft), $I_{B}=1.630\left[\mathrm{~kg} \mathrm{~m}^{2}\right]$ (float), the length along the keel from step to center of gravity $l_{c g}=0.104[\mathrm{~m}]$, the distance of CG from the keel $v_{c g}=0.453[\mathrm{~m}]$, and the moment arm length from CG to the supports (Figure 5) are $l_{A f}=l_{A b}=l_{B f}=l_{B b}=0.2[\mathrm{~m}]$. Froude number is defined based on the beam length $F_{n_{B}}=U / \sqrt{g B}$. The beam length $B$ and $l_{c g}$ are not reported in Hirakawa's paper ${ }^{8}$ and their values are educated guesses. In the simulation, we use the half body representation. That means we half the mass and moment of inertia in the calculations, and model as a mono-hull float plane.

For plots with vertical axis showing the maximum eigenvalue $\Re(\sigma)_{\max }$ of the linear system, any positive value of the real part of the eigenvalue signifies divergence of the oscillation mode and therefore is unstable. The eigenvalues are calculated from the matrix obtained in the following way. $\mathbf{K}=\mathbf{M}^{-1}(-\mathbf{R})$, where $\mathbf{M} \dot{x}+\mathbf{R} x=0$ and $x$ is the state vector, where $\mathbf{M}$ and $\mathbf{R}$ represent the matrices in equation (1) and equation (2).

Figure 7 confirms that moving the CG backwards towards the step helps the craft to remain stable until a higher velocity. The figure shows the maximum real part of the eigenvalues $\Re(\sigma)_{\max } \sqrt{B / g}$ with respect to Froude number $F_{n_{B}}$. We kept the beam length $B$ constant. Thus, Froude number is essentially a non-dimensionalized speed. In the towing tank experiment, divergence (porpoising) occurred at about $U=5.0[\mathrm{~m} / \mathrm{s}]^{8}\left(F_{n_{B}}=3.57\right)$ with the nominal $l_{c g} / B$ location of 5.2. Figure 7 shows that, in the numerical simulation, planing craft with $l_{c g} / B=0.50$ turns unstable at just under $F_{n_{B}}=5.0$ and with $l_{c g} / B=0.65$, just under $F_{n_{B}}=3.5$. Note that in the physical dimensions, the two $l_{c g}$ values differ only by $0.03[\mathrm{~m}]$ (or $1.95 \%$ of the float length of $1.54[\mathrm{~m}]^{8}$ ) and the speed limit for stable planing changed by $2.10[\mathrm{~m} / \mathrm{s}]$ (or $42.8 \%$ difference). Thus, the planing speed $U$ at which the craft turns unstable is very sensitive to the $\mathrm{CG}$ location. Considering the fact that values for $B$ and $l_{c g}$ are only approximately known, the numerical results are very reasonable in light of the experimental evidence.

The trim angles $\tau$ corresponding to the two $l_{c g}$ values with respect to $F_{n_{B}}$ are shown in Figure 8. The trim angles are found by driving the moment equation to have near zero residue moment. This is done using Brent's method [16, Ch.3-4] implemented in Scipy optimize module of the Python programming language. One can let the solutions to have 
small residues so that the trim angles found can be used as a small perturbation in the subsequent eigenvalue computations.

Figure 9 indicates that the desirable direction of moving the CG ,i.e. forward or backward, to stabilize an unstable planing condition depends on the current value of $l_{c g}$. There is a band of $l_{c g}$ values at which a non-decaying oscillation manifests with positive $\Re(\sigma)_{\max } \sqrt{B / g}$. This band of instability increases in width as $F_{n_{B}}$ increases from 2.86 to 5.71 as seen in Figure $9 \mathrm{a}$ to Figure 9c. The contour plots show that the sensitivity of the stability to changes in $v c g$ is not as marked as changes in $l_{c g}$. A small portion of the design space near the transom or very small value of $l_{c g}$ generates stable designs, and most float planes have this configuration to facilitate the pitch up at the moment of take off. This means that to make the planing stable, it is a good idea to shift the CG aft. However, once airborne, it is better to have CG forward to have enough "static margin" for a stable flight.

Figure 10 checks whether the two simulation codes, one for the rigid-body case and the other for the flexibly supported case, agree if the flexible support's spring were extremely stiff. The plot shows $\Re(\sigma)_{\max } \sqrt{B / g}$ with respect to $F_{n_{B}}$. The two lines agree very well.

Figure 11 - Figure 13 show the effectiveness of the flexible support in mitigating unstable oscillations. However, as can be noted from the sharp rise in the real part of eigenvalues at high Froude numbers, it is not a globally stabilizing solution. Inadequate damping in the flexible supports can worsen the stability of the seaplane compared with the conventional rigidly supported ones as seen in Figure 11a or in Figure 11b. This suggests that the damper should be designed carefully in such a way that no divergent oscillation modes occur in the planing speeds of the aircraft. Figure 12 shows that if only one of either front or back support is made flexible, it is the back support that is effective in mitigating instabilities. Similarly, if damping is applied to either the front or the back support (that are both flexible), it is the damping of the back support that is more effective (Figure 13). These results show some similarity with the flutter stability phenomena in Aeroelasticity, in which the elastic axis location of the wing affects the divergence speed.

Figure 14 shows a contour plot of $\Re(\sigma)_{\max } \sqrt{B / g}$ with respect to $l_{c g}$ and $v c g$ along with sampled points by SOMBAS in two-design-variable case. SOMBAS was set to search feasible designs requiring $\Re(\sigma)_{\max }<0$. The sampled points that satisfies that condition are shown along with the final location of the training samples for Self-Organizing Map (SOM). The distribution of the training samples indicates the finite sample representation of the feasible region around which further sampling in the subsequent iterations are expected produce further space filling effect of the feasible design space, i.e. further stable designs. In this trivial case (because we already have the contour plot), we see that SOMBAS sampled diverse combinations of $l_{c g}$ and $v_{c g}$ filling out the stable domain. This feasible region search capability is useful when the design space is in higher dimensions (many design variables) 
and full-factorial design (or grid sampling) becomes too expensive.

Figure 15a shows the scatter plot matrix of the seven design variable case. $N_{f}$ is the number of designs (experiments) computed by SOMBAS and $N_{s}$ is the number designs that satisfy the condition, i.e., $\Re(\sigma)_{\max }<0$. The lower triangular cells show the absolute values of correlation coefficients. Again, it clearly shows the unstable "band" for $l_{c g}$ at the top row of the scatter plot matrix. Other parameter does not show clear unfeasible regions. Further restriction was applied by setting $\Re(\sigma)_{\max }<-0.3$ and the results are shown in Figure 15b. It shows some new trends. For example, $v c g$ tends to lower value as the eigenvalue becomes more negative. On the other hand the beam length $B$ tends to larger value as the eigenvalue becomes more negative. The $l_{c g}$ concentrates between 0.6 and 1.1, and $v c g$ tends to low values as $l_{c g}$ becomes longer.

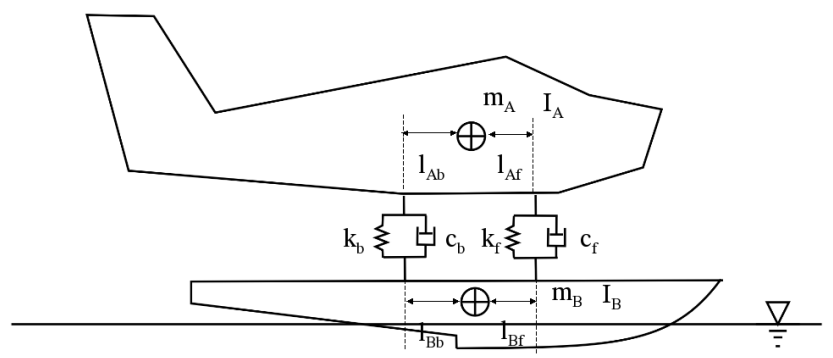

Figure 5: Schematic of a float plane with the flexible support

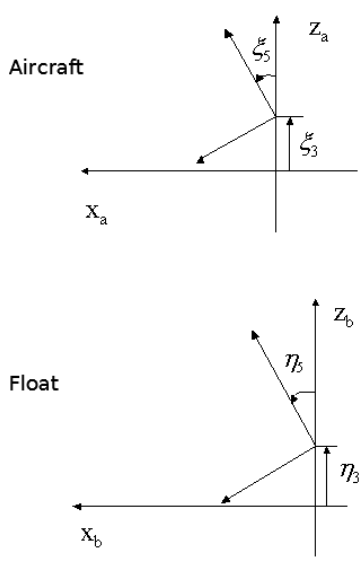

Figure 6: Coordinate system for the Small Perturbation Method 


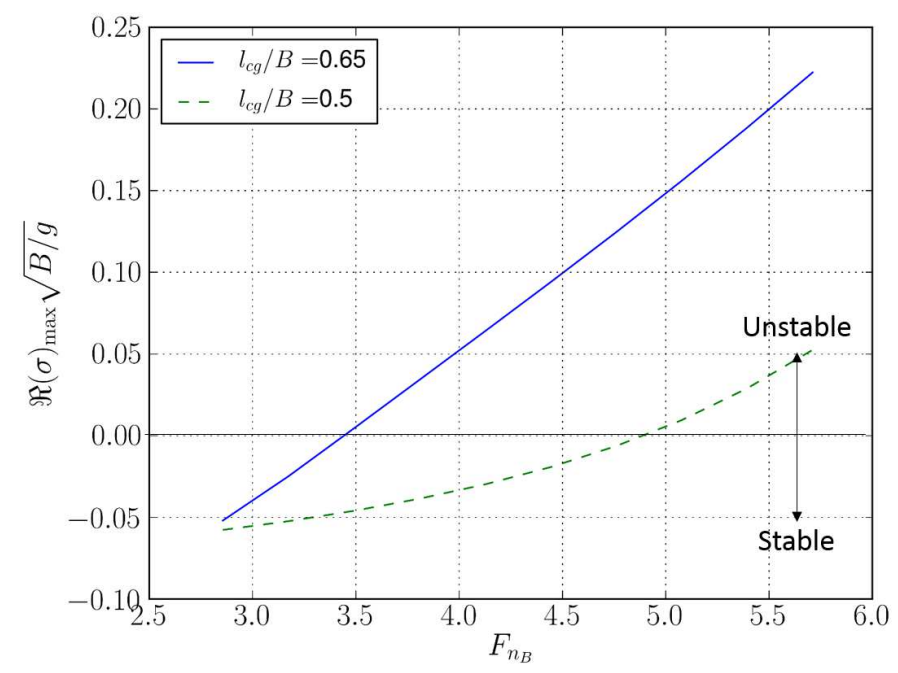

Figure 7: Porpoising mitigation by moving the CG aft.

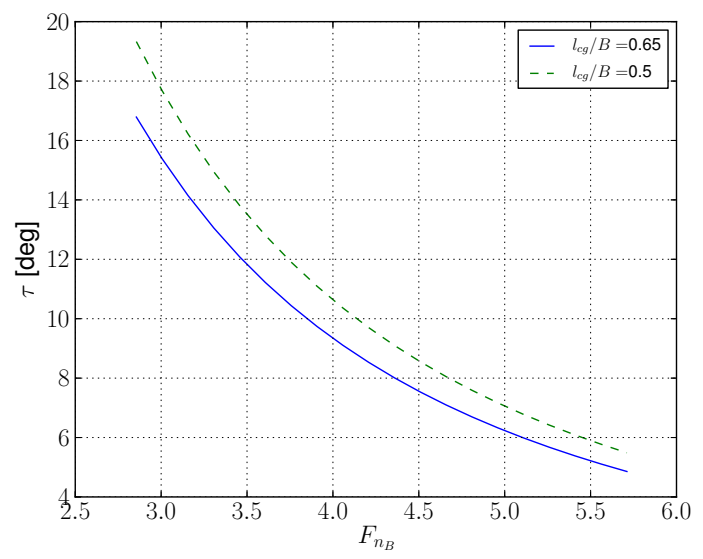

Figure 8: Trim angle of the simulated model with respect to Froude number $F_{n_{B}}$ 


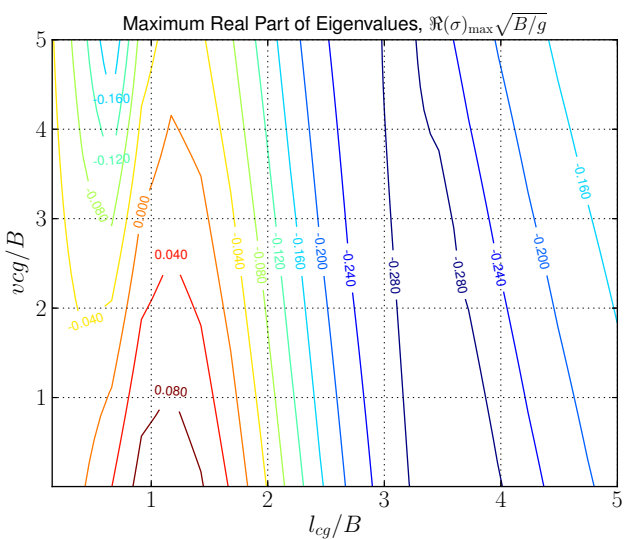

(a) $F_{n_{B}}=2.86$

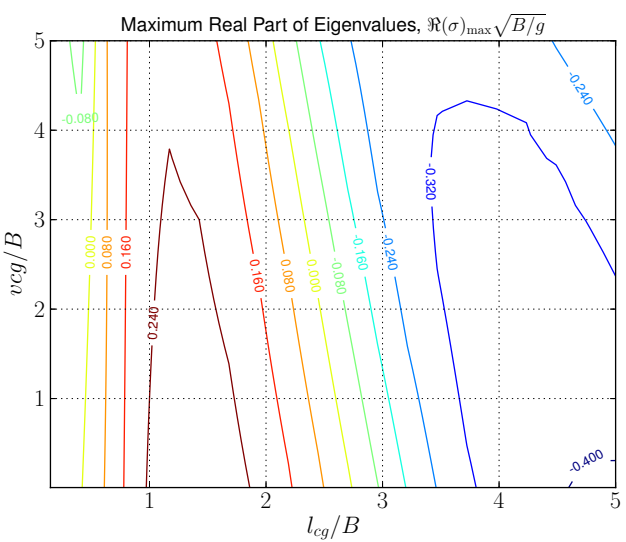

(b) $F_{n_{B}}=4.28$

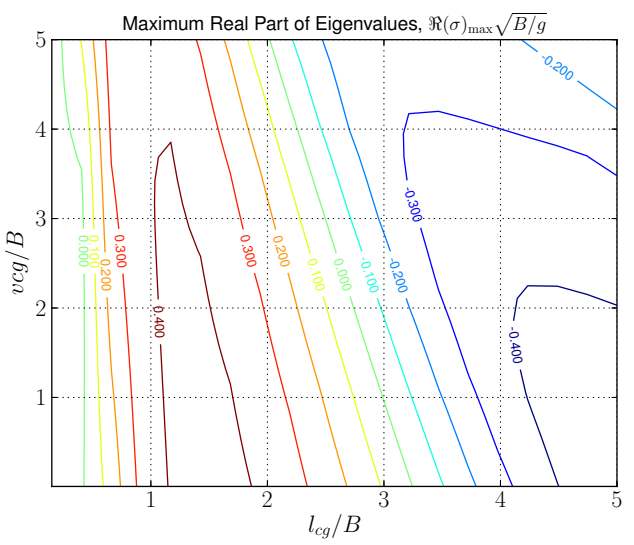

(c) $F_{n_{B}}=5.71$

Figure 9: Stability with respect to $l_{c g}$ and $v_{c g} . \Re(\sigma)_{\max } \sqrt{B / g}<0$ are stable designs.

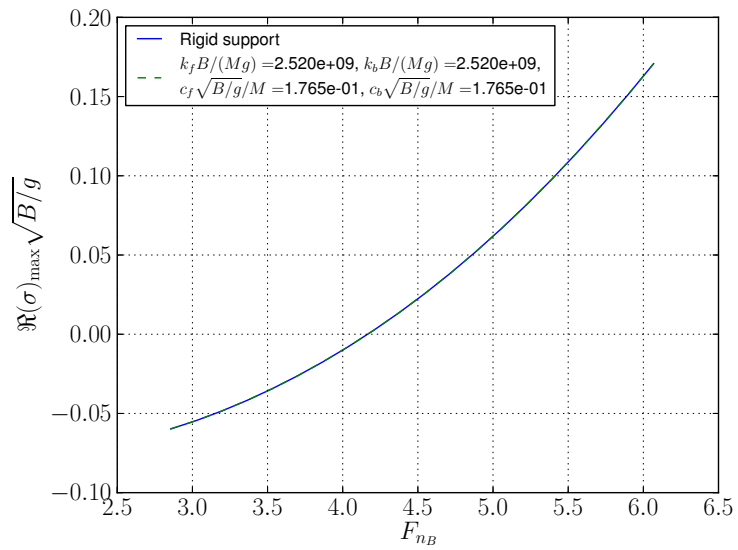

Figure 10: Comparison of stability results in the rigid-body formulation and the flexiblesupport formulation with very stiff springs 


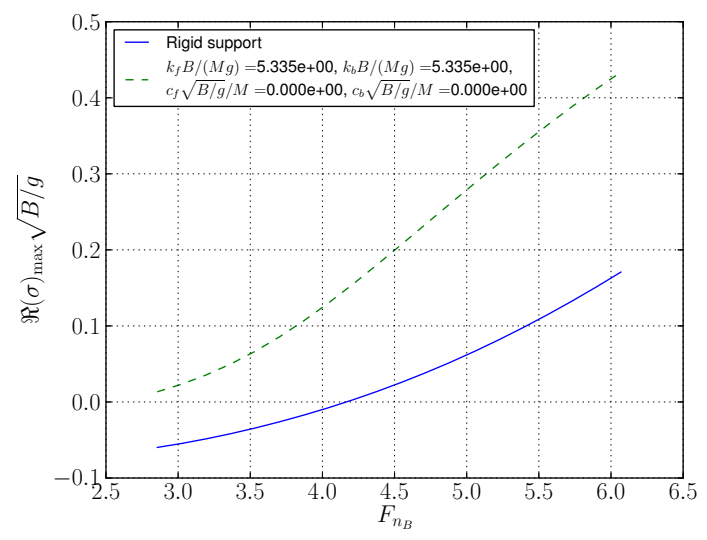

(a) No damping

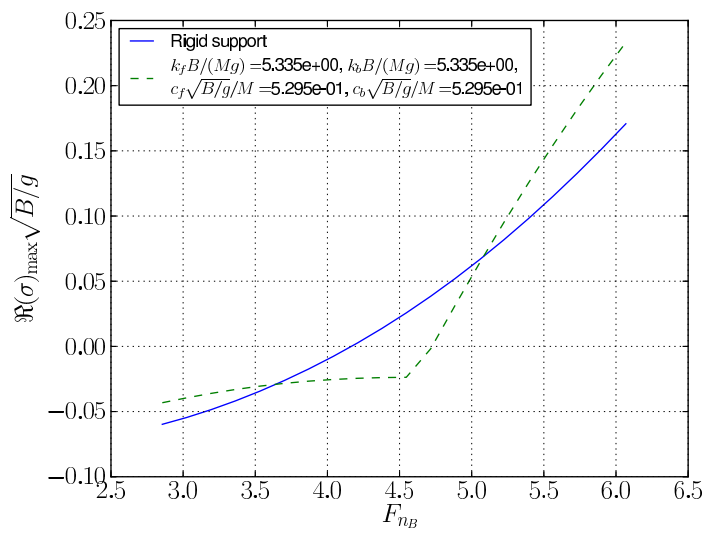

(c) $c_{f, b}=30[N s / m]$

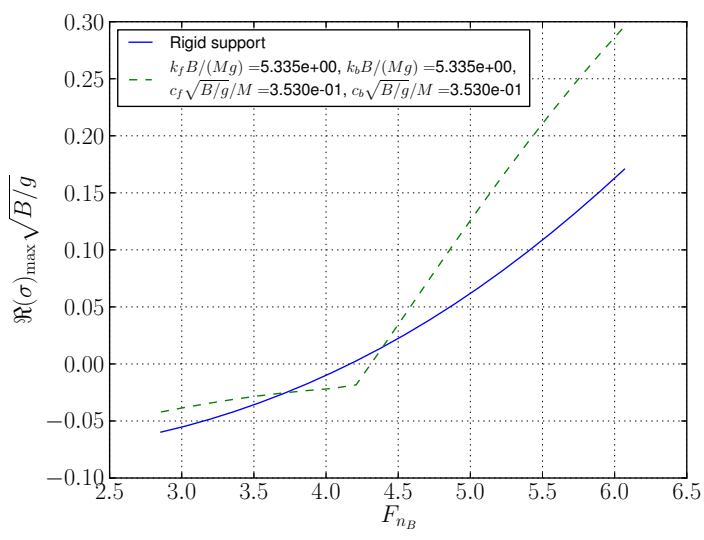

(b) $c_{f, b}=20[N s / m]$

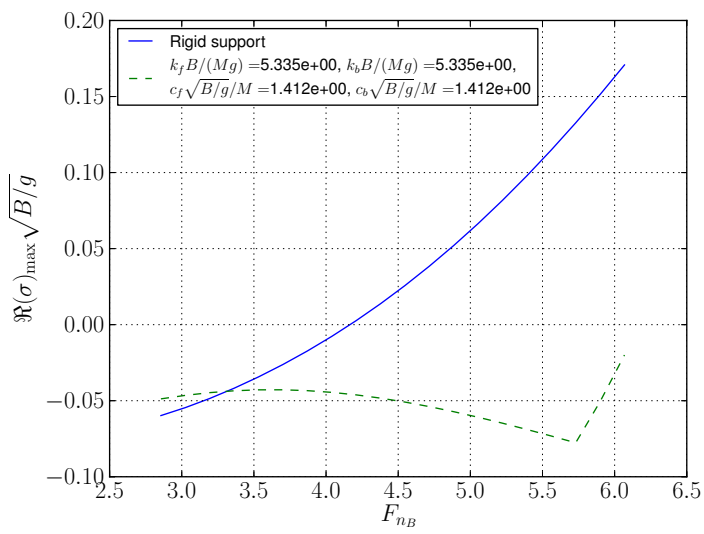

(d) $c_{f, b}=80[N s / m]$

Figure 11: Comparison of longitudinal stability between rigidly supported case and flexibly supported case. Fixed spring constant $k_{f, b}=2117[\mathrm{~N} / \mathrm{m}]$ with various damping coefficients. 


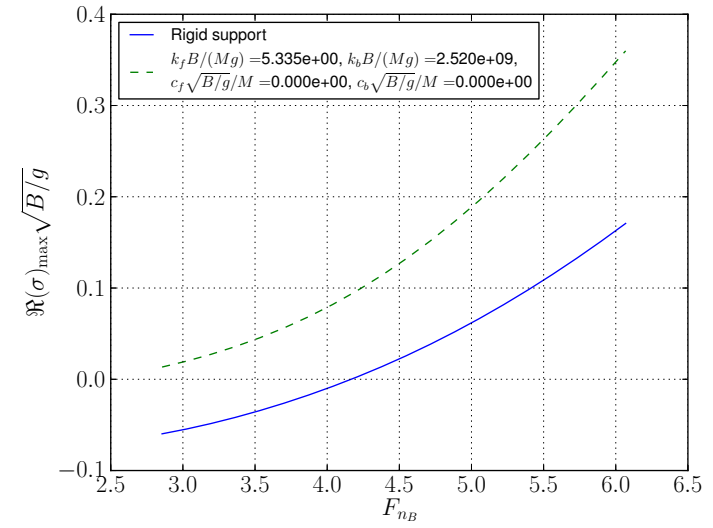

(a) Flexible front support, no damping

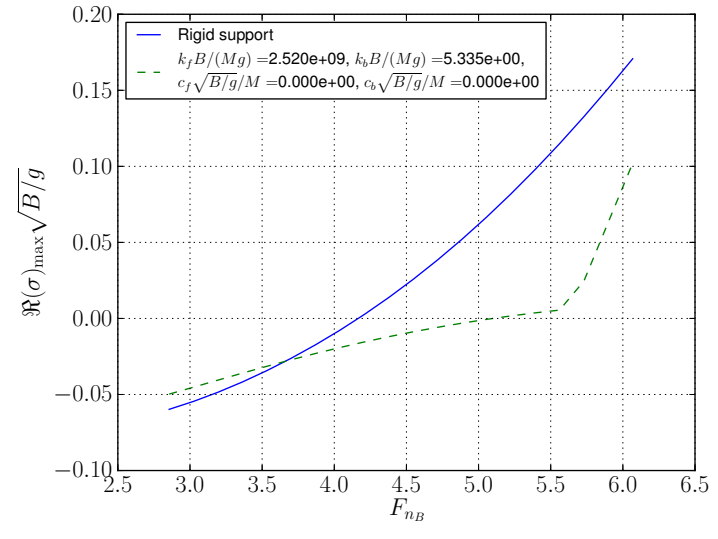

(b) Flexible back support, no damping

Figure 12: Comparison of longitudinal stability between the rigidly supported case and the flexibly supported case. Spring applied only to the front $k_{f}$ or to the back $k_{b}$, with no damping applied

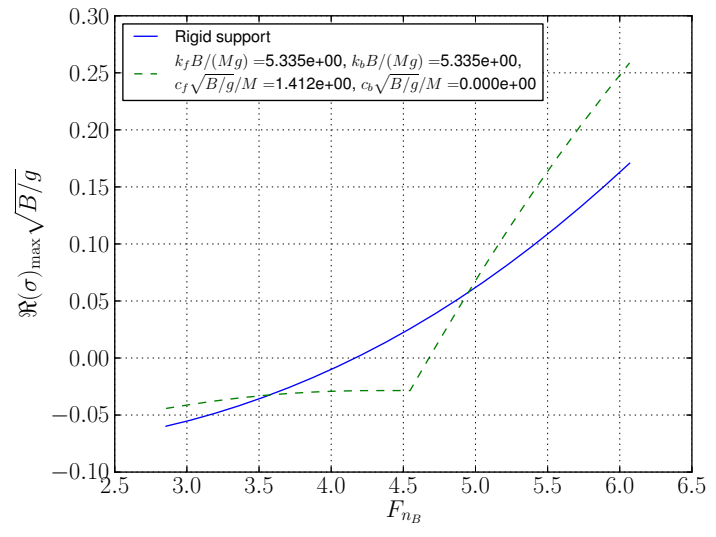

(a) Applying damping only to the front support

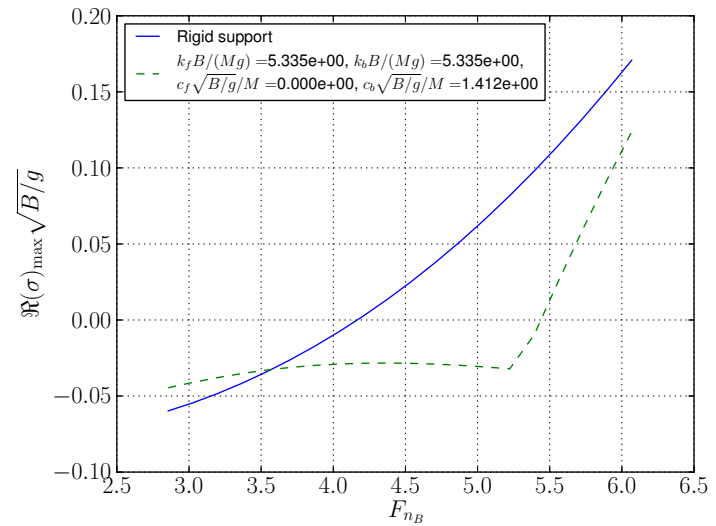

(b) Applying damping only to the back support

Figure 13: Comparison of longitudinal stability between the rigidly supported case and the flexibly supported case. Fixed spring constant $k_{f, b}=2117[\mathrm{~N} / \mathrm{m}]$, with damping of $80[\mathrm{~N} \mathrm{~s} / \mathrm{m}]$ applied to either the front or the back support 


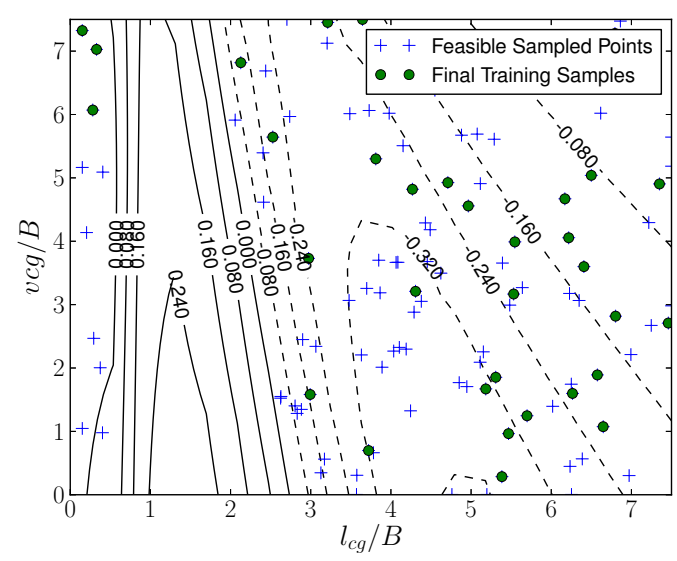

Figure 14: SOMBAS sampling stable combinations of $l_{c g}$ and $v c g$ at $F_{n_{B}}=4.28$

\section{Discussions}

It is desirable to conduct further experiments to improve the quality of the model by calibration and further numerical model refinement. The hull geometry and the flexiblesupport model employed in this research were very simple. Inclusion of the afterbody of the float (the portion after the step) may create another planing surface at larger pitch angles, and this may create another instability. Further sophistication in the flexible-support model, including their control may render further insights and new opportunities.

In the current study, the CG location $\left(l_{c g}\right.$ and $\left.v c g\right)$ and the pitching moment of inertia $\left(I_{55}, I_{A, B}\right)$ were treated independently. However, in reality, they are not. If you change the CG location, so do the moments of inertia. Thus, care must be taken to interpret the results in this paper where both variables are treated independently.

In this paper the aerodynamic effects were not considered. However, seaplanes receive substantial lift force at planing speeds and the elevator provides a means to control the pitch angle. Thus the aerodynamics may have a substantial effect on the planing characteristics of a seaplane. The inclusion of the aerodynamic effects will be the next step in the development of the numerical simulation of the planing seaplanes.

Active and semi active control of the flexible support ${ }^{17}$ can be considered to obtain a desired dynamics in both waveless and wavy conditions. For example Skyhook methods in which damping is controlled to emulate the dynamics of bodies whose damper is attached to an imaginary inertial frame (hence Skyhook) could be employed. This may be effective in eliminating porpoising as well as improving operational reliability in wavy conditions. Of course, it comes at the cost of added weight and complexities.

Once validated, the parametric models (flexible or rigid) equip us with the means to conduct various trade-off studies concerning porpoising at early design stages. These should 

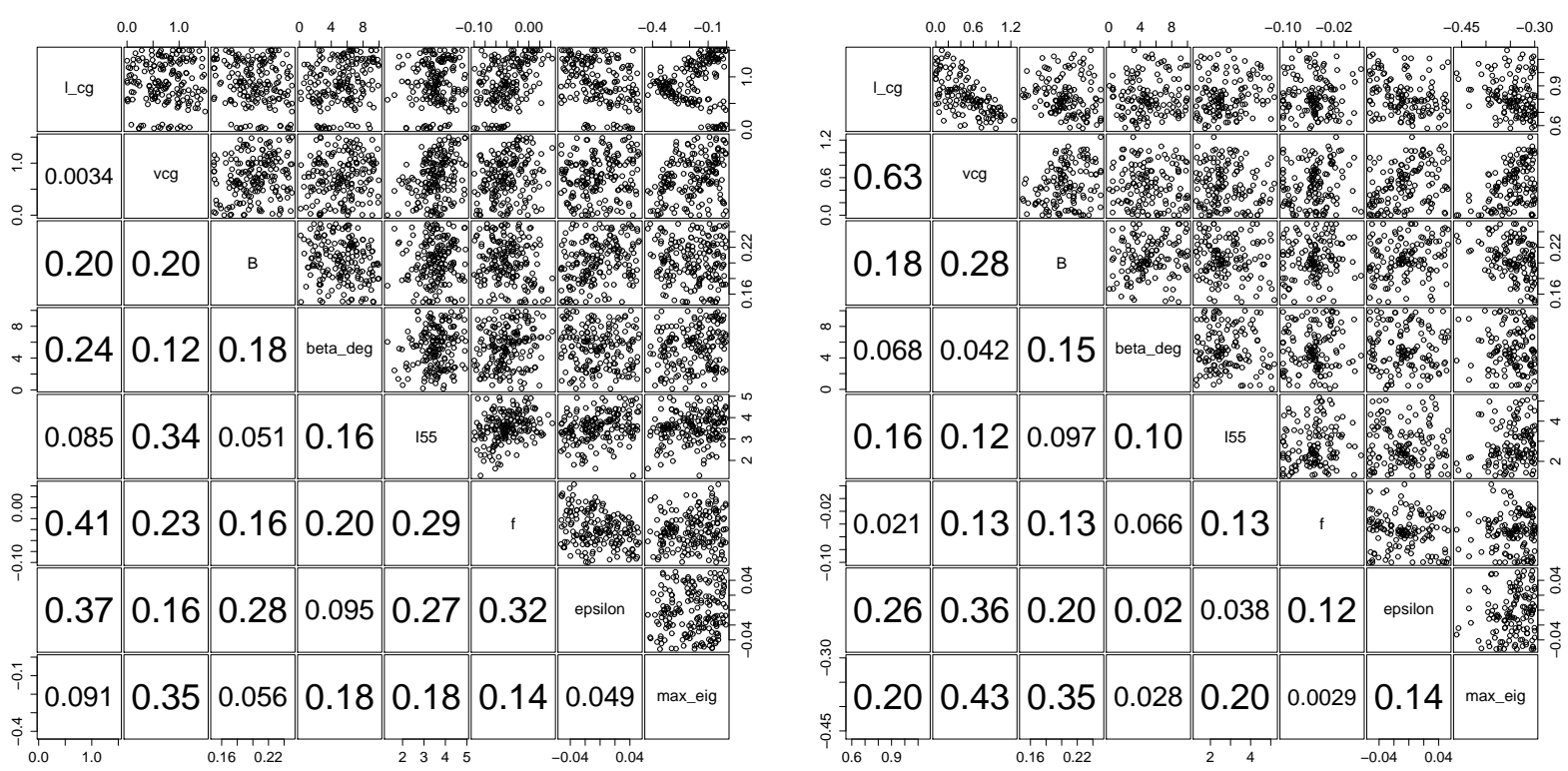

(a) Maximum eigenvalues of oscillation modes (b) Maximum eigenvalues of oscillation modes less than $0,\left(N_{f}=289, N_{s}=181\right)$

less than $-0.3,\left(N_{f}=504, N_{s}=135\right)$

Figure 15: Scatter Matrix showing distribution of feasible designs at $F_{n_{B}}=4.28$

provide more informed design decisions than the rules of thumbs hitherto available. With the inclusion of aerodynamic forces, they will be useful in assessing the sizing, location and incidence angles of the lifting surfaces with respect to planing hulls, and whether the flexible support would be needed. Eventually, a new set of design standards could be drawn concerning longitudinal stability of waterborne seaplanes including the design of the flexible support.

\section{Conclusion}

The numerical analysis of the Linear-Time-Invariant model revealed useful information to the questions posed. The computation shows that whether one should move the center of gravity backward or forward will depend on the position of center of gravity with respect to the step of the planing hull. If a flexible support is employed one may postpone the inception of porpoising to a much higher Froude number. The simulation results indicate that damping coefficients in the flexible supports play an important role and the range of planing speed will determine their values. The damping in the hind support was more effective than the damping in the front support. These numerical results can be used to conduct more dedicated physical experiments to quantitatively assess the numerical models and confirm physical phenomena. Furthermore, aerodynamic effects must be taken into account. These 
will constitute the future work of this study along with the parametric optimization of the system.

\section{Acknowledgment}

Keiichi Ito has been funded by the Institute for the Promotion of Innovation through Science and Technology (IWT) through the Baekeland Mandate program in the research and development of SOMBAS. His research has also been funded by the Interuniversity Attraction Poles Programme BESTCOM initiated by the Belgian Science Policy Office. The undergoing project of developing innovative seaplanes (project managed by Tatsumi Sakurai and the members of Hiyoh Aircraft Manufacturing and Development Co. and Aeromarine Systems Cooperation Association) is an endeavour supported by many dedicated and enthusiastic volunteers.

\section{Appendix}

\section{A. Ovreview of Hydrodynamic Calculations}

The computation of stability takes two steps. First, the equilibrium or the trim position needs to be found given the planing speed. Second, the small perturbation analysis is performed about the equilibrium position by looking at the increment in force and moment with respect change in state vector variables. As in Flight Dynamics, the stability derivatives need to be obtained about this equilibrium point. We will follow closely the procedure given in Falinsen. ${ }^{10} \mathrm{~A}$ big part of the following is due to Savitsky ${ }^{18}$ and Wagner. ${ }^{19}$

\section{Trim Determination}

For trim determination, the following equilibria hold. Vertical forces,

$$
M g=N \cos \tau+T \sin (\tau+\epsilon)-R_{v} \sin \tau
$$

Horizontal forces,

$$
T \cos (\tau+\epsilon)=R_{v} \cos \tau+N \sin \tau
$$

Pitching moments,

$$
N c+R_{v} a-T f=0 .
$$


The $N$ represents the resultant hydrodynamic pressure force (represented to be acting at a point along and perpendicular to the keel line in the vertical $x z$ plane). The $T$ is the thrust force from the power plant. These two forces can be expressed with respect to $\tau$, and $R_{v}$ using the equations (3) and (4) ( $\epsilon$ is given by the design). The $R_{v}$ is the frictional force exerted on the planning surface. Then these can be plugged into the moment force equation (5) to obtain an equation of $a, c, \tau$, and $R_{v}$ ( $f$ is given by the design),

$$
\frac{c}{\cos \tau}\left(M g-\frac{M g \sin \tau+R_{v}}{\cos \epsilon} \sin (\tau+\epsilon)+R_{v} \sin \tau\right)+R_{v} a-\frac{M g \sin \tau+R_{v}}{\cos \epsilon} f=0
$$

The frictional force is given by

$$
R_{v}=\frac{1}{2} \rho C_{F} S(U \cos \tau)^{2} \simeq \frac{1}{2} \rho C_{F} S U^{2}
$$

where $\rho$ is water density, $S$ is the wetted surface area and $C_{F}$ is the friction coefficient.

$$
C_{F}=\frac{0.075}{\left(\log _{10}\left(R_{n}\right)-2\right)^{2}}+\frac{44\left[\left(\frac{A H R}{L_{K}}\right)^{1 / 3}-10 R_{n}^{-1 / 3}\right]+0.125}{10^{3}} .
$$

where $A H R$ is the average hull roughness in micrometers. We set to $A H R=150[\mu m]$. The $R_{n}$ is the Reynolds number based on the characteristic length $L_{K}$ which is the wetted keel length. The wetted area $S$ is given as following.

$$
S=\frac{\tan ^{2} \beta}{\sin \beta} \frac{B^{2}}{4\left(1+\frac{z_{\max }}{V t}\right) \tau}+\frac{B}{\cos \beta} L_{C},
$$

where $1+\frac{z_{\max }}{V t} \simeq \pi / 2$ for small $\beta$ (for large $\beta$, see $[10, \mathrm{p} .303]$ ), and $L_{C}$ is the wetted chine length. Note that $R_{v}$ is a function of $\tau, L_{K}$ and $L_{C}$ which we don't know. The two moment arm lengths $a$ and $c$ pertaining to the hull frictional force $R_{v}$ and hydrodynamic pressure force $N$ respectively can be formulated as following,

$$
\begin{aligned}
a & =v c g-\frac{B}{4} \tan (\beta), \\
c & =l_{c g}-l_{p} .
\end{aligned}
$$

The $a$ is fixed given CG location, $B$, and $\beta$, but to get $c$, we need to determine the distance of center of hydrodynamic pressure from the step $l_{p}$. To determine $l_{p}$, we take three steps. First, we determine $C_{L_{0}}$ using

$$
C_{L_{\beta}}=\frac{F_{L_{\beta}}}{0.5 \rho U^{2} B^{2}}=C_{L_{0}}-0.0065 \beta_{\text {deg }} C_{L_{0}}^{0.60}
$$


where $F_{L_{\beta}}=M g$. Second, given $F_{n_{B}}$ we can obtain $\lambda_{W}$ from

$$
C_{L_{0}}=\frac{F_{L_{0}}}{0.5 \rho U^{2} B^{2}}=\tau_{d e g}^{1.1}\left[0.012 \lambda_{W}^{0.5}+0.0055 \frac{\lambda_{W}^{2.5}}{F_{n_{B}}^{2}}\right]
$$

Again, we do not know the value for $\tau_{d e g}$. Thus, we temporarily assume some value for $\tau_{d e g}$. Third, the following equation gives $l_{p}$.

$$
\frac{l_{p}}{\lambda_{W} B}=0.75-\frac{1}{\frac{5.21 F_{n_{B}}^{2}}{\lambda_{W}^{2}}+2.39} .
$$

With the $\lambda_{W}$ computed using assumed $\tau=\pi \tau_{\text {deg }} / 180$, we can also obtain $L_{K}$ and $L_{C}$ from

$$
\begin{aligned}
\lambda_{W} & =\frac{L_{K}+L_{C}}{2 B}, \\
\frac{B}{2} & =\frac{\pi}{2 \tan \beta}\left(L_{K}-L_{C}\right) \tau .
\end{aligned}
$$

These enable us to compute $R_{v}$ for the assumed $\tau$. We now check if equation (6) holds. We iterate the process using Brent's method until we find a good $\tau$.

\section{Stability Derivatives}

Based on the trim condition obtained for a given planing speed $U$, we can now proceed to the calculation of the stability derivatives. The restoring force coefficients due to heave $C_{33}$ and pitch $C_{35}$ are given by

$$
\begin{aligned}
& \frac{C_{33}}{0.5 \rho U^{2} B}=-\left.B \frac{\partial C_{L_{\beta}}}{\partial \eta_{3}}\right|_{0}=-\left.B \frac{\partial C_{L_{0}}}{\partial \eta_{3}}\right|_{0}\left[1-0.0039 \beta_{d e g} C_{L_{0}}^{-0.4}\right] \\
& \frac{C_{35}}{0.5 \rho U^{2} B}=-\left.B \frac{\partial C_{L_{\beta}}}{\partial \eta_{5}}\right|_{0}=-\left.B \frac{\partial C_{L_{0}}}{\partial \eta_{5}}\right|_{0}\left[1-0.0039 \beta_{d e g} C_{L_{0}}^{-0.4}\right]
\end{aligned}
$$

where $\left.\right|_{0}$ denotes "at the static equilibrium position" and

$$
\begin{aligned}
\left.\frac{\partial C_{L_{0}}}{\partial \eta_{3}}\right|_{0}= & \left.\tau_{\operatorname{deg}}^{1.1}\left[0.006 \lambda_{0}^{-0.5}+\frac{0.01375 \lambda_{0}^{1.5}}{F_{n_{B}}^{2}}\right] \frac{\partial \lambda_{W}}{\partial \eta_{3}}\right|_{0}, \\
\left.\frac{\partial C_{L_{0}}}{\partial \eta_{5}}\right|_{0}= & 1.1\left(\frac{180}{\pi}\right)^{1.1} \tau^{0.1}\left[0.012 \lambda_{0}^{0.5}+\frac{0.0055 \lambda_{0}^{2.5}}{F_{n_{B}}^{2}}\right] \\
& +\left.\tau_{\operatorname{deg}}^{1.1}\left[0.006 \lambda_{0}^{-0.5}+\frac{0.01375 \lambda_{0}^{1.5}}{F_{n_{B}}^{2}}\right] \frac{\partial \lambda_{W}}{\partial \eta_{5}}\right|_{0}
\end{aligned}
$$


Here, $\lambda_{0}$ is the value of $\lambda_{W}$ at the static equilibrium. Furthermore,

$$
\begin{aligned}
\left.\frac{\partial \lambda_{W}}{\partial \eta_{3}}\right|_{0} & =-\frac{1}{\sin \tau} \frac{1}{B} \\
\left.\frac{\partial \lambda_{W}}{\partial \eta_{5}}\right|_{0} & =\frac{-\operatorname{vcg} / B}{\sin ^{2} \tau}+\frac{z_{w l} / B}{\sin ^{2} \tau} \cos \tau+\frac{0.25 \tan \beta}{\left(1+z_{\max } / V t\right) \tau^{2}}
\end{aligned}
$$

From equation (14), we can write

$$
\left.\frac{1}{B} \frac{\partial l_{p}}{\partial \lambda_{W}}\right|_{0}=0.75-\frac{15.63 \frac{F_{n_{B}}^{2}}{\lambda_{0}^{2}}+2.39}{\left(5.21 \frac{F_{n_{B}}^{2}}{\lambda_{0}^{2}}+2.39\right)^{2}} .
$$

Then, we can compute the pitch moment coefficients due to heaving $C_{53}$ and due to pitching $C_{55}$ as

$$
\begin{aligned}
\frac{C_{53}}{0.5 \rho U^{2} B^{2}} & =-\left[\frac{1}{B} \frac{\partial l_{p}}{\partial \lambda_{W}} B \frac{\partial \lambda}{\partial \eta_{3}} C_{L_{\beta}}+\left(\frac{l_{p}}{B}-\frac{l_{c g}}{B}\right) B \frac{\partial C_{L_{\beta}}}{\partial \eta_{3}}\right]_{0}, \\
\frac{C_{55}}{0.5 U^{2} B^{3}} & =-\left[\frac{1}{B} \frac{\partial l_{p}}{\partial \lambda_{W}} \frac{\partial \lambda_{W}}{\partial \eta_{5}} C_{L_{\beta}}+\left(\frac{l_{p}}{B}-\frac{l_{c g}}{B}\right) \frac{\partial C_{L_{\beta}}}{\partial \eta_{5}}\right]_{0} .
\end{aligned}
$$

The added mass calculations are divided into two hull regions: the region where the keel is wet but the chine is not (the triangular planform region) and the region thereafter up to the step where the planing surface is completely wet (the rectangular planform region). Strip theory is used to exploit two-dimensional analytical solutions. A two-dimensional added mass of a wedge can be given using Gamma function,

$$
a_{33} \equiv \rho d^{2} K=\frac{\rho d^{2}}{\tan \beta}\left[\frac{\pi}{\sin \beta} \frac{\Gamma\left(1.5-\frac{\beta}{\pi}\right)}{\Gamma^{2}\left(1-\frac{\beta}{\pi}\right) \Gamma\left(0.5+\frac{\beta}{\pi}\right)}-1\right]
$$

where $d$ is the draft and we only consider the real part of $K$. The $d$ is defined as the draft from the spray root position,

$$
d=\left(1+\frac{z_{\max }}{V t}\right) x \tau
$$

Here, $x$ is on the axis that increases towards the step along the keel line with origin at the point where the wetting of keel starts. In particular, we denote $x_{s}$ as the point at which chine wetting starts. The added mass in the heaving of the triangular region is obtained from

$$
A_{33}^{(1)}=\rho K\left(1+\frac{Z_{\max }}{V t}\right) \tau^{2} \int_{0}^{x_{s}} x^{2} d x
$$

where

$$
x_{s}=\frac{B}{2} \frac{\tan \beta}{\left(1+\frac{Z_{\max }}{V t}\right) \tau}
$$


After integration, we have

$$
\frac{A_{33}^{(1)}}{\rho B^{3}}=\frac{K}{24} \frac{\tan ^{3} \beta}{\left(1+\frac{Z_{\max }}{V t}\right) \tau} .
$$

For the pitch induced added mass in the triangular region,

$$
A_{35}^{(1)}=A_{53}^{(1)}=-\rho K \int_{0}^{x_{s}} d^{2}\left(x-x_{G}\right) d x
$$

where $x_{G}=L_{K}-l_{c g}$. After inegration, we obtain

$$
\frac{A_{35}^{(1)}}{\rho B^{4}}=\frac{A_{53}^{(1)}}{\rho B^{4}}=\frac{A_{33}^{(1)}}{\rho B^{3}} \frac{x_{G}}{B}-\frac{K}{64} \frac{\tan ^{4} \beta}{\left(1+\frac{Z_{\max }}{V t}\right)^{2} \tau^{2}} .
$$

We also have

$$
A_{55}^{(1)}=\rho K \int_{0}^{x_{s}} d^{2}\left(x-x_{G}\right)^{2} d x
$$

which results in

$$
\frac{A_{55}^{(1)}}{\rho B^{5}}=\frac{K}{160} \frac{\tan ^{5} \beta}{\left(1+\frac{Z_{\max }}{V t}\right)^{3} \tau^{3}}-\frac{K}{32} \frac{x_{G}}{B} \frac{\tan ^{4} \beta}{\left(1+\frac{Z_{\max }}{V t}\right)^{2} \tau^{2}}+\left(\frac{x_{G}}{B}\right)^{2} \frac{A_{33}^{(1)}}{\rho B^{3}} .
$$

As for the rectangular region, the heaving induced part is given by

$$
\frac{A_{33}^{(2)}}{\rho B^{3}}=C_{1} \frac{\pi}{8} \frac{L_{C}}{B}
$$

where

$$
C_{1}=\frac{2 \tan ^{2} \beta}{\pi} K
$$

The pitching induced added masses of the rectangular portion are

$$
\frac{A_{35}^{(2)}}{\rho B^{4}}=\frac{A_{53}^{(2)}}{\rho B^{4}}=-C_{1} \frac{\pi}{16}\left[\left(\frac{L_{K}}{B}\right)^{2}-\left(\frac{x_{s}}{B}\right)^{2}\right]+\frac{x_{G}}{B} \frac{A_{33}^{(2)}}{\rho B^{3}}
$$

and

$$
\frac{A_{55}^{(2)}}{\rho B^{5}}=\frac{C_{1} \pi}{24}\left(\left(\frac{L_{K}}{B}\right)^{3}-\left(\frac{x_{s}}{B}\right)^{3}\right)-\frac{C_{1} \pi}{8}\left(\frac{x_{G}}{B}\right)\left(\left(\frac{L_{K}}{B}\right)^{2}-\left(\frac{x_{s}}{B}\right)^{2}\right)+\left(\frac{x_{G}}{B}\right)^{2} \frac{A_{33}^{(2)}}{\rho B^{3}}
$$

The resulting added masses are given by $A_{i j}=A_{i j}^{(1)}+A_{i j}^{(2)}$.

The damping coefficients $B_{i j}$ are analysed using a quasi-steady approach in which changes in the angle of attack immediately cause changes in lift. We exclude hydrostatic effects in 
equations (12) and (13) by setting $F_{n_{B}} \rightarrow \infty$.

$$
C_{L_{\beta}}=C_{L_{0}}-0.0065 \beta_{\text {deg }} C_{L_{0}}^{0.60}
$$

where

$$
C_{L_{0}}=\left(\frac{180}{\pi}\right)^{1.1} \tau^{1.1} 0.012 \lambda_{W}^{0.5} .
$$

Because of the heave velocity, there is a change in angle of attack (trim)

$$
\alpha=-\frac{d \eta_{3}}{d t} / U=-\frac{\dot{\eta}_{3}}{U}
$$

This causes a vertical force

$$
F_{3}=-\frac{1}{2} \rho U^{2} B^{2} \frac{\partial C_{L_{\beta}}}{\partial \tau} \frac{\dot{\eta}_{3}}{U}
$$

where

$$
\frac{\partial C_{L_{\beta}}}{\partial \tau}=\frac{\partial C_{L_{0}}}{\partial \tau}\left[1-0.0039 \beta_{\text {deg }} C_{L_{0}}^{-0.4}\right]
$$

and

$$
\frac{\partial C_{L_{0}}}{\partial \tau}=0.0132\left(\frac{180}{\pi}\right)^{1.1} \tau^{0.1} \lambda_{W}^{0.5} .
$$

Note the $\dot{\eta}_{3}$ in equation (42). This gives the heaving damping force,

$$
\frac{B_{33}}{\rho B^{3}\left(\frac{g}{B}\right)^{\frac{1}{2}}}=0.5 F_{n_{B}} \frac{\partial C_{L_{\beta}}}{\partial \tau} .
$$

The pitching moment resulting from the heave velocity can be computed using

$$
F_{5}=F_{3}\left(0.75 \lambda_{W} B-l_{c g}\right)
$$

and this gives

$$
\frac{B_{53}}{B_{33} B}=0.75 \lambda_{W}-\frac{l_{c g}}{B} .
$$

The damping coefficients $B_{55}$ and $B_{35}$ are studied with respect to $d \eta_{5} / d t,{ }^{20}$ and given as

$$
\begin{gathered}
B_{55}=U x_{T}^{2} a_{33}\left(x_{T}\right), \\
B_{35}=U A_{33}-U x_{T} a_{33}\left(x_{T}\right) .
\end{gathered}
$$

where

$$
a_{33}\left(x_{T}\right)=\rho\left(\frac{B \tan \beta}{2}\right)^{2} K
$$


is the two dimensional added mass in heave for the cross section at step $x_{T}=l_{c g}$.

\section{References}

${ }^{1}$ Darrol Stinton. Aero-marine design and flying qualities of floatplanes and flying-boats. Aeronautical Journal, pages 97-127, March 1987.

${ }^{2}$ John B. Parkinson. Appreciation and determination of the hydrodynamic qualities of seaplanes. Technical Note 1290, National Advisory Committee for Aeronautics, Washington, May 1947.

${ }^{3} \mathrm{Jr}$. Charles L. Shuford. A theoretical and experimental study of planing surfaces including effects of cross section and plan form. Report 1355, National Advisory Committee for Aeronautics, 1958.

${ }^{4}$ H. M. Garner. Porpoising test on a model of a flying boat hull. Reports and Memoranda 1492, Marine Aircraft Experimental Establishment, March 1932.

${ }^{5}$ K. M. Tomaszewski. Hydrodynamic design of seaplane floats. Current papers, Ministry of Supply Aeronautical Research Council, 1950.

${ }^{6}$ Dale De Remer. Water Flying Concepts: an Advanced Text on Wilderness Water Flying. Aviation Supplies \& Academics, Newcastle, Washington, 2nd edition, 1990.

${ }^{7}$ Burke Mees. Notes of a Seaplane Instructor. Aviation Supplies \& Academics, Newcatle, Washington, 1998.

${ }^{8}$ Yoshiaki Hirakawa, Takehiko Takayama, Asuka Kosaki, Hiromitsu Kikuchi, Tsugukiyo Hirayama, and Tatsumi Sakurai. Model experiment of a suppression-system for wave impact and porpoising phenomena. Conference Proceedings of The Japan Society of Naval Architects and Ocean Engineers (in Japanese), 3:239$242,2006$.

${ }^{9}$ Alexander Klemlin, John D. Pierson, and Edmund M. Storer. An introduction to seaplane porpoising. Journal of the Aeronautical Sciences, 6(8):1905-1915, June 1939.

${ }^{10}$ Odd M. Faltinsen. Hydrodynamics of High-Speed Marine Vehicles. Cambridge University Press, 2005.

${ }^{11}$ Serge Abrate. Hull slamming. Applied Mechanics Reviews, 64(6):060803-1 - 060803-35, November 2011.

${ }^{12}$ Yoshiho Ikeda and Toru Katayama. Porpoising oscillation of very-high-speed marine craft. Philosophical Transactions of The Royal Society, Series A, 358(1771):1905-1915, 2000.

${ }^{13}$ James M. Benson and Anton Freihofner. Methods and charts for computing stability derivatives of a vbottom planing surface. Technical Report NACA ARR 3L08, National Advisory Committee for Aeronautics, Langley Memorial Aeronautical Laboratory, 1943.

${ }^{14}$ Keiichi Ito, Tom Dhaene, Naji El Masri, Roberto d'Ippolito, and Joost Van de Peer. Selforganizing map based adaptive sampling. In Proceedings of 5th International Conference on Experiments/Process/System Modeling/Simulation/Optimization (5th IC-EpsMsO), volume II, pages 504 - 513, Athens, Greece, July 3 - 6 2013. ISBN:978-618-80527-2-7 or 978-618-80527-0-3.

${ }^{15}$ Keiichi Ito, Ivo Couckuyt, Roberto d'Ippolito, and Tom Dhaene. Design space exploration using Self-Organizing Map based adaptive sampling. Applied Soft Computing, 2016. Accepted.

${ }^{16}$ Richard P. Brent. Algorithms for Minimization Without Derivatives. Prentice-Hall, Englewood Cliffs, NJ, 1973.

${ }^{17}$ M. Canale, M. Milanese, and C. Novara. Semi-active suspension control using "fast" model-predictive techniques. Control Systems Technology, IEEE Transactions on, 14(6):1034-1046, Nov 2006. 
${ }^{18}$ Daniel Savitsky. Hydrodynamic design of planing hulls. Marine Technology, 1(1):71 - 96, October 1964.

${ }^{19}$ Herbert Wagner. Über stoß- und gleitvorgänge an der oberfläche von flüssigkeiten. Zeitschrift für Angewandte Mathematik und Mechanik, 12(4):193 - 215, August 1932. In German.

${ }^{20}$ Nils Salvesen, E.O. Tuck, and Odd Faltinsen. Ship motions and sea loads. SNAME Transactions, 78:250-287, 1970. 